\title{
Tipificaciones en especies sudamericanas de HEDEOMa, LEPECHINIA Y STACHYS (LAMIACEAE) Y NUEVO SINÓNIMO EN LEPECHINIA
}

\author{
NATALY O'LEARY1
}

\begin{abstract}
Resumen: Se designan ocho lectotipos para los siguientes nombres pertenecientes a especies de Lamiaceae: Astemon graveolens Regel, Hedeoma adscendens Rusby, Hedeoma mandonianum Wedd., Lepechinia floribunda (Benth.) Epling, Lepechinia sagittata Epling, Lepechinia vesiculosa (Benth.) Epling, Sphacele hastata Griseb. y Stachys albicaulis Lindl. A su vez se propone a Lepechinia sagittata Epling como nuevo sinónimo de Lepechinia floribunda (Benth.) Epling.
\end{abstract}

Palabras clave: Lamiaceae, tipificación, taxonomía.

Summary: Typifications in South American species of Hedeoma, Lepechinia and Stachys (Lamiaceae) and new synonym in Lepechinia. Eight lectotypes are here designated for the following names belonging to Lamiaceae species: Astemon graveolens Regel, Hedeoma adscendens Rusby, Hedeoma mandonianum Wedd., Lepechinia floribunda (Benth.) Epling, Lepechinia sagittata Epling, Lepechinia vesiculosa (Benth.) Epling, Sphacele hastata Griseb. and Stachys albicaulis Lindl. Furthermore, Lepechinia sagittata Epling is proposed as a new synonym for Lepechinia floribunda (Benth.) Epling.

Key words: Lamiaceae, typification, taxonomy.

\section{INTRODUCCIÓN}

En el contexto de la revisión de la familia Lamiaceae para el proyecto Flora Argentina, (Anton \& Zuloaga, 2015), se encontraron varios taxones pertenecientes a los géneros Hedeoma Pers., Lepechinia Willd. y Stachys L., representados en Argentina cada uno por tres especies válidas, que poseen problemas de tipificación. En este trabajo se procede a la resolución de las tipificaciones de ocho taxones, a través de la designación de lectotipos, y se propone un nuevo sinónimo.

\section{Materiales y Métodos}

Los protólogos de todos los nombres tipificados

\footnotetext{
${ }^{1}$ Instituto de Botánica Darwinion, Labardén 200, c.c. 22, B1642HYD, San Isidro, Buenos Aires, Argentina. E-mail. noleary@darwin.edu.ar
}

(Astemon graveolens Regel, Hedeoma adscendens Rusby, Hedeoma mandonianum Wedd., Lepechinia floribunda (Benth.) Epling, Lepechinia sagittata Epling, Lepechinia vesiculosa (Benth.) Epling, Sphacele hastata Griseb. y Stachys albicaulis Lindl.) han sido examinados, y los especímenes tipo han sido localizados en los siguientes herbarios: CGE, CORD, F, GOET, K, LA, LE, NY, P y UC, por medio del acceso en línea a la página de JSTOR y/o la comunicación personal con los curadores de dichos herbarios. Cuando el ejemplar posee código de barras se indica el número del mismo seguido de las iniciales "cb".

Es importante remarcar las recientes aclaraciones de McNeill (2014), dónde entre otras observaciones se explica que según el Artículo 40, nota 1 (McNeill et al., 2012) solo se puede asegurar que un material es un holotipo si se está seguro de que el autor del nombre no usó ningún otro material y no existe ningún otro duplicado del mismo, sea en diferentes herbarios o en un mismo herbario. 


\section{Resultados}

Se propone la designación de ocho lectotipos y se justifica la elección de los mismos.

Hedeoma mandonianum Wedd., Chlor. Andina 2: 148. 1860. Tipo: Bolivia. [Dpto. La Paz] Cordillère de Sorata, 1858, Mandon 116 (lectotypus aquí designado $\mathrm{P}$ cb 00714577 !). Nota 1 .

Hedeoma adscendens Rusby, Phytologia 1: 74: 1934. Tipo: Bolivia. [Dpto. La Paz] Top of pass on the Tipuani-ancoma-Sorata, 30-V-1926, Tate 783 (lectotypus aquí designado NY cb 00000575 !; isolectotipo NY cb 00000576!). Nota 2.

Nota 1. Slanis y Bulacio (2005: 285) señalan que el holotipo de Hedeoma mandonianum estuvo depositado en $\mathrm{B}$ y que ha sido probablemente destruido. Sin embargo, a partir del protólogo no es posible asegurar que el material original hubiera estado depositado en B. En relación con esto, H. A. Weddell realizó sus estudios en Francia, en el Museo de Historia Natural del París (P) (Stafleu \& Cowan, 1988) y probablemente haya estudiado el material coleccionado por Mandon allí. Por tal motivo, se propone como lectotipo el especimen depositado en P.

Nota 2. En NY, donde se encuentran los materiales de especies descriptas por H. Rusby en sus últimos años, junto con las colecciones de G. H. H. Tate (Stafleu \& Cowan, 1983) se encontraron dos hojas de herbario de la colección "Tate 783", mencionada en el protólogo y que se ajustan a éste. Estas hojas no poseen referencia cruzada como pertenecientes a un mismo ejemplar, por lo tanto, y de acuerdo al CIN (Art. 40, nota 1) (McNeill et al., 2012) es apropiado seleccionar a uno de ellos como lectotipo. Se designa aquí, como lectotipo de $H$. adscendens, a la hoja con código de barras 00000575. Esta es la más completa en cuanto al estado del material; la restante hoja (NY cb 00000576) resulta un isolectotipo.

Lepechinia floribunda (Benth.) Epling, Repert. Spec. Nov. Regni Veg. Beih. 85: 22. 1935. Sphacele floribunda Benth., Prodr. 12: 254. 1848. Tipo: "H. Hook. Ex hort. Berol.” Sphacele floribunda (lectotypus aquí designado $\mathrm{K} \mathrm{cb}$ 484020!). Nota 3.

Sphacele hastata Griseb., Abh. Königl. Ges. Wiss. Göttingen 19: 238. 1874, hom. illeg. (non A. Gray, 1862). Alguelagum grisebachii Kuntze, Revis. Gen. Pl. 2: 512. 1891, nom. nov. Sphacele grisebachii Kurtz, Revista Mus. La Plata 5: 294. 1893, nom. nov. Tipo: Argentina. Córdoba, pr. Las Peñas, freq. in convalle Arroyo, II-1871, Lorentz 142 (lectotypus aquí designado GOET cb 004265!; isolectotipo, CORD cb 6146!). Nota 4.

Lepechinia sagittata Epling, Bull. Torrey Bot. Club 71: 487. 1944. Tipo: Argentina. Córdoba, Colón, de Ascochinga a La Cumbre, 13-XII-1938, Goodspeed s.n. (lectotypus aquí designado UC cb 657352!). Syn. nov. Nota 5 .

Nota 3. Epling (1935: 22) realiza la combinación bajo Lepechinia, y menciona que vio en Kew el ejemplar tipo. Siguiendo las recomendaciones en el trabajo de McNeill (2014), donde se establece la necesidad de lectotipificar siempre que se tenga duda sobre el tipo, se procede a designar lectotipo el ejemplar localizado en $\mathrm{K}$.

Nota 4. A. T. Hunziker (1960), en su publicación sobre los tipos "grisebachianos" depositados en CORD señala que el ejemplar Lorentz 142 (en CORD) corresponde a un "isotipo", por cuanto este autor suponía que el holotipo estaría conservado en GOET. Aunque aclara que en aquel momento no tuvo acceso a los ejemplares de Lorentz en GOET (sí lo tuvo en años posteriores). Por ese motivo se procede aquí a la designación del lectotipo, siguiendo las recomendaciones de McNeill (2014) donde se aclara que la certeza sobre si un material es holotipo sólo se puede tener si el autor así lo estableció explícitamente, caso contrario se trata de un lectotipo.

Tanto O. Kuntze (1891) como dos años después Kurtz (1893) quisieron darle un nombre nuevo al taxón Sphacele hastata Griseb., el cual era ilegítimo por ser un homónimo posterior de $S$. hastata A. Gray. Ambos autores le asignaron el epíteto "grisebachii", en honor a Grisebach, pero en diferentes géneros: Alguelagum grisebachii Kuntze y Sphacele grisebachii Kurtz. 
Epling, propone la sinonimia de Sphacele hastata bajo Lepechinia floribunda y señala que no pudo encontrar el ejemplar tipo de este taxon.

Nota 5. Epling (1944) indica en el protólogo de L. sagittata que el ejemplar tipo proviene de una planta crecida en Los Ángeles (Estados Unidos de América) a partir de semillas coleccionadas en Argentina por T. Goodspeed, y que el mismo fue depositado en la Universidad de California. Andrew Doran (com. pers., UC) encontró un ejemplar en ese herbario que se corresponde con los caracteres dados en el protólogo y que es aquí designado como lectotipo.

A partir del estudio de este material se ha podido constatar que Lepechinia sagittata se corresponde morfológicamente con Lepechinia floribunda, siendo que posee las flores en pseudo racimos o glomérulos axilares o terminales y las hojas de lámina deltoide-ovada, características distintivas de Lepechinia floribunda, y por lo tanto aquí se propone ubicarlo en sinonimia de este último.

Lepechinia vesiculosa (Benth.) Epling, Repert. Spec. Nov. Regni Veg. Beih. 85: 24. 1935. Hyptis vesiculosa Benth., Labiat. Gen. Spec.: 131. 1833. Sphacele vesiculosa (Benth.) J. F. Macbr., Field Mus. Nat. Hist. Bot. Ser. 13(5): 769. 1960. Tipo: [Ecuador] Perú [sin localidad, 1778-1788], Tafalla s.n. [herb. H. Ruiz \& J. A. Pavón]. (lectotypus aquí designado F, fotografía de la Serie Field Museum, número de negativo 17802 ! ex $\mathrm{B}^{\dagger}$ ). Nota 6 .

Astemon graveolens Regel, Index Sem. (St. Petersburg) 38-39. 1860. Lepechinia graveolens (Regel) Epling, Repert. Spec. Nov. Regni Veg. Beih. 85: 25. 1935. Tipo: Bolivia. Cochabamba, semina misit Regel, Ex horto bot. Petropolitano 599 (lectotypus aquí designado LE cb 00004789 !; isolectotipo LA cb 00000111!). Nota 7.

Nota 6. En 1784 Gómez Ortega, director del Real Jardín Botánico de Madrid, designó a Juan José Tafalla como agregado en la expedición científica de Hipólito Ruiz y José Pavón. Los ejemplares colectados por Tafalla en Perú quedaron incluidos en la colecciones de Ruiz y Pavón (Muñoz Garmendía, 2004) y algunos de ellos fueron adquiridos por el botánico inglés A. B.
Lambert entre 1816 y 1824 . Luego de su muerte, el "Herbario Lambertiano" fue dividido en 137 partes y vendido a 16 compradores diferentes. La colección de Ruiz y Pavón fue vendida en al menos 3 lotes y adquiridos, en primera instancia, por R. Brown (BM) y W. Pamplin (OXF), y luego, algunos especímenes pasaron a otros herbarios europeos (B, G, por ejemplo) y estadounidenses (MO, NY, US) (Miller, 1970).

Adicionalmente, se buscaron posibles duplicados del material tipo entre las colecciones de Ruiz \& Pavón en el herbario MA (Stafleu \& Cowan, 1983). Entre éstas se encontraron 5 ejemplares identificados como Sphacele vesiculosa pero ninguno de ellos posee referencias a Tafalla ni menciona la localidad tipo, Guayaquil. Por estos motivos, no es posible asegurar que estos ejemplares constituyan material original.

Epling (1935: 24) señaló haber visto un probable ejemplar tipo en Kew. En dicho herbario se localizó un ejemplar con determinavit de Epling del año 1928, que dice "probable isotype of Hyptis vesiculosa Benth" (K cb 000485335). La etiqueta en dicho ejemplar sólo indica "Dracocephalum de Peru, sp. nova", sin hacer referencia al colector, localidad ni fecha de colecta. Otro determinavit, de R. M. Harley del año 1987 sobre este especimen, dice "This is apparently not the type of $L$. vesiculosa (Benth.) Epling”.

Ante la ausencia de un ejemplar original se designa como lectotipo la fotografía (Art. 9.2, McNeill et al., 2012). En la etiqueta del lectotipo seleccionado (fotografía ex B) se lee "In Peruvia Tafalla". Sin embargo probablemente se trate del actual territorio de Ecuador siendo ue en la época de la colección todo era conocido como Perú.

Nota 7. Según Stafleu y Cowan (1983) los materiales de E. A. von Regel están depositados en el herbario LE. En este herbario se encontró un ejemplar que posee en letra manuscrita la inscripción "semina misit Regel index seminum 38-39. 1860" lo cual concuerda con el protólogo. Por lo tanto, se designa al mismo como lectotipo de Astemon graveolens. En el herbario LA se encontró un fragmento del mencionado ejemplar de LE, el cual constituye un isolectotipo.

Stachys albicaulis Lindl., Edwards's Bot. Reg. 18: 1558. 1833. Tipo: Chile. Collina, 1825, Mc Rae 
s.n. (lectotypus aquí designado CGE cb 06740!; isolectotipos $\mathrm{K}$ cb 000485351 !, $\mathrm{K}$ cb 000485349!). Nota 8.

Nota 8. En el protólogo se lee: "The late Mr. McRae first found it in Chile at the baths of Collina, a favourite retreat of the inhabitants of St. Jago. Since that time it has been met with by others, and is probably not an uncommon Chilian Species". Según Stafleu y Cowan (1981) el Herbario de Lindley está en CGE (Cambridge), incluyendo sus tipos, y también hay algunos ejemplares en BM y K. Por esto seguramente Epling en 1927 colocó "isotype" en las etiquetas de los ejemplares de K.

\section{Agradecimientos}

A los curadores de los herbarios mencionados en el texto por su ayuda en la localización de material tipo, principalmente a Lesley Walsigham $(\mathrm{K})$ y Christine Bartram (CGE). La presente contribución, en su versión original fue corregida y mejorada por M. Belgrano (SI) a quien también se le agradece la dedicación. Este trabajo se encuentra financiado por el proyecto CONICET PIP 00537/13.

\section{Biblografía}

ANTON, A. \& F. O. ZULOAGA (directores). http:// www.floraargentina.edu.ar/ [Acceso: 2 junio 2015].

EPLING, C. C. 1935-37. Synopsis of the Southamerican Labiatae. Repert. Spec. Nov. Regni Veg. Beih. 85: 1-341.

EPLING, C. C., 1944. Lamiaceae. En: Moldenke, H. (ed.), Contributions to the Flora of Extratropical South America. Lilloa 10: 348-352.

HUNZIKER, A. T. 1960. Catálogo de los tipos "grisebachianos" conservados en Córdoba. Bol. Acad. Nac. Ci. 41: 283-421
JSTOR. Global Plants. http://plants.jstor.org/. [Acceso: 1 octubre 2014].

KUNTZE, O. 1891. Labiatae. En: Revisio Generum Plantarum 2,pp. 511-531. A. Felix, Leipzig.

KURTZ, F. 1893. Sertum Cordobense. Revista Mus. La Plata 5: 282-303.

MC NEILL, J., F. R. BARRRIE, W. R. BUCK, V. DEMOULIN, W. GREUTER, D. L. HAWKSWORTH, P. S. HERENDEEN, S. KNAPP, K. MARHOLD, J. PRADO, W. F. PRUD'HOMME VAN REINE, G. F. SMITH, J. H. WIERSEMA \& N. J. TURLAND. 2012. International Code Nomenclature (Melbourne Code). Reg. Veg. 154. Königstein: Koeltz Scientific Books.

MCNEILL, J. 2014. Holotype specimens and type citations: General issues. Taxon 63: 1112-1113.

MILLER, H. S. 1970. The herbarium of Aylmer Bourke Lambert: Notes on its acquisition, dispersal, and present whereabouts. Taxon 19: 489-656.

MUÑOZ GARMENDÍA, F. 2004. La botánica al servicio de la Corona. La Expedición de Ruiz, Pavón y Dombey al virreinato del Perú (1777-1831). Barcelona, Lunwerg.

PONTIROLI, A. 1993. Labiatae. En: Cabrera, A. L. (ed.), Flora de la provincia de Jujuy. Colecc. Ci. Inst. Nac. Tecnol. Agropecu. 13(9): 117-155.

SLANIS, C. A. \& E. BULACIO. 2005. Hedeoma mandonianum (Lamiaceae) en Argentina. Bol. Soc. Argent. Bot. 40: 285-287.

STAFLEU, F. A. \& R. S. COWAN. 1981. Taxonomic literature Vol. 3. Bohn, Scheltema \& Holkema, Utrecht.

STAFLEU, F. A. \& R. S. COWAN. 1983. Taxonomic literature Vol. 4. Bohn, Scheltema \& Holkema, Utrecht.

STAFLEU, F. A. \& R. S. COWAN. 1988. Taxonomic literature Vol. 7. Bohn, Scheltema \& Holkema, Utrecht.

Recibido el 20 de enero de 2015, aceptado el 12 de marzo de 2015. 